\title{
A Case of Myxedema Coma Confounded by Dementia and Hypokalemia
}

\author{
Jake Namjik Cho ${ }^{\text {a, b, d, Stephen Avera }}{ }^{\text {a, b }}$, Kenneth Iyamu ${ }^{\text {a, c }}$
}

\begin{abstract}
The hallmarks of myxedema coma are decreased mental status and hypothermia; but hypotension, bradycardia, hyponatremia, hypoglycemia, and hypoventilation may also be present. This case involves a 74-year-old female, with arthritis and dementia, who presented with weakness and failure to thrive. Physical exam was significant for altered level of consciousness, lethargy, pretibial pitting edema and hypotension. Since friends and family were absent, history was obtained per chart review. Initial labs showed severe hypokalemia of 2.0 $\mathrm{mmol} / \mathrm{L}$ and associated changes of prolonged QT interval and T wave flattening on electrocardiogram (ECG). The family later revealed additional history of thyroidectomy and clarification that the patient had been noncompliant with her medications for at least 6 months. Hypothyroidism was confirmed with a thyroid stimulating hormone (TSH) level of $82.2 \mathrm{U} / \mathrm{mL}$ and a free thyroxine (T4) level below normal threshold. Endocrinology was consulted and care was taken when reinstituting her thyroid supplementation after checking cortisol level as well as reviewing her ECGs. This case report illustrates the importance of checking for thyroid dysfunction especially in the elderly who present with altered mental status and unclear history as most symptoms of myxedema coma may be reversible. Furthermore, myxedema coma occurring in the setting of decompensated hypothyroidism is a medical emergency. In this case, despite timely diagnosis and appropriate treatment, her cognitive function failed to improve, and as a result, palliative care was consulted. Ultimately, the family elected for hospice care due to severe dementia, bed-bound status, and failure to thrive.
\end{abstract}

Keywords: Myxedema coma; Hypothyroid; Levothyroxine; Cortisol; Encephalopathy; Failure to thrive

\section{Introduction}

Myxedema coma is defined as severe hypothyroidism leading

Manuscript submitted May 24, 2019, accepted June 7, 2019

aUniversity of Central Florida College of Medicine, Graduate Medical Education, Orlando, FL, USA

${ }^{b}$ Internal Medicine Residency Program, Ocala Regional Medical Center, Ocala, FL, USA

'Internal Medicine Faculty, Ocala Regional Medical Center, Ocala, FL, USA ${ }^{\mathrm{d} C}$ Corresponding Author: Jake Namjik Cho, 2810 SW 16th Place, Ocala, FL 34474, USA.Email: Jake.Cho@ucf.edu

doi: https://doi.org/10.14740/jem574 to decreased mental status, hypothermia, and other metabolic imbalances [1]. A common misconception is that a patient must be comatose to be diagnosed with myxedema coma. Myxedema refers to the swelling of the skin and soft tissue that occurs in patients who are hypothyroid. Myxedema coma occurs in the setting of decompensated hypothyroidism usually precipitated by some factor such as infection [1]. The hallmark of myxedema coma is a deterioration of the patient's mental status [1]. Although myxedema coma is exceedingly rare with about 200 cases reported between 1953 and 1996 [1], and an incidence of about 0.22 million per year, more common in women and the elderly [2], myxedema coma is a medical emergency. If not promptly diagnosed and treated, the mortality rate can be more than $50 \%$, with the most common causes of death being respiratory failure, sepsis, and gastrointestinal bleeding [3].

\section{Case Report}

The patient is a 74-year-old female, with past medical history of arthritis and dementia, who presents with weakness and failure to thrive. Upon initial evaluation, patient was lethargic and with difficulty staying awake. She was oriented to self only and uncooperative with the interview as she would selectively answer some questions and repeat her desire to go home. Past medical history was unobtainable as family could not be reached at the time of evaluation. Emergency department records indicated that patient was brought by ambulance by request of the husband who stated that patient has not been eating, drinking or bathing for 2 weeks. Patient admitted to poor oral intake of food and water of 1 month duration. Initial workup revealed severe hypokalemia. Physical exam was significant for altered mental status (AMS) and abnormal insight, lethargy, pretibial pitting edema (Fig. 1) and hypotension.

With critically low potassium level of $2.0 \mathrm{mmol} / \mathrm{L}$, prolonged corrected QT interval of $633 \mathrm{~ms}$ (Fig. 2) and metabolic alkalosis (Table 1), electrolytes replacement was initiated. Further history obtained from the family the next day revealed history of thyroidectomy and that patient has had AMS, lethargy, anorexia and medication noncompliance for 6 months prior. Given this additional history, severe hypothyroidism was suspected and confirmed with a thyroid stimulating hormone (TSH) level of $82.2 \mathrm{U} / \mathrm{mL}$ and a free thyroxine (T4) level below test threshold $(0.07 \mathrm{ng} / \mathrm{dL})$. AM cortisol level was normal at $20.60 \mu \mathrm{g} / \mathrm{dL}$ (Table 1). Initial dose of $300 \mu \mathrm{g}$ intravenous 


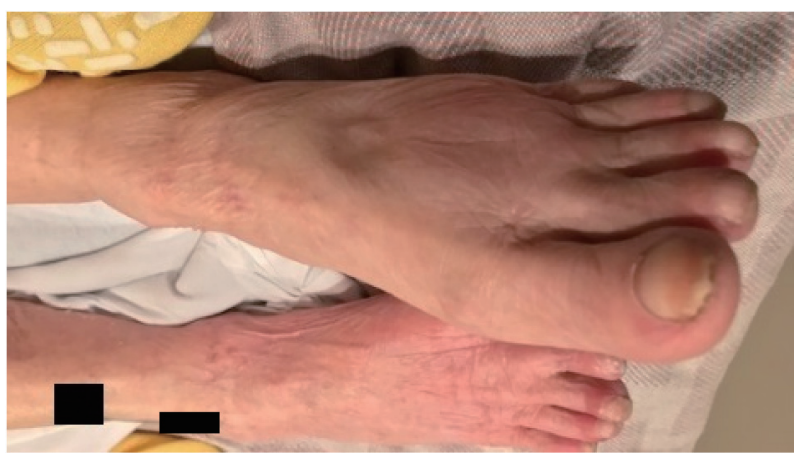

Figure 1. Pretibial pitting edema.

(IV) levothyroxine (T4) and $10 \mu \mathrm{g}$ IV triiodothyronine (T3) were administered after consultation with endocrinology. This was subsequent dosing of T4 $50 \mu \mathrm{g}$ IV daily and T3 at $5 \mu \mathrm{g}$ IV for one additional dose.

Prolonged QTc improved to $414 \mathrm{~ms}$ and free T4 improved to $1.22 \mathrm{ng} / \mathrm{dL}$. Despite 5 days of supportive care, her cognitive function failed to improve, and as a result, palliative and hospice care consultations were sought as requested by family due to severe dementia, bed-bound status, and failure to thrive (Fig. 3).

\section{Discussion}

This case presents a unique instance of severe hypothyroidism and myxedema coma. Clinical findings of elevated TSH and low T4/T3 (primary hypothyroidism), myxedema coma are usually associated with hyponatremia as a result of decreased free water clearance. This is due to either elevated antidiuretic hormone or diminished renal perfusion. Hypoglycemia may be seen as a result of down-regulation of metabolism or adrenal insufficiency. Arterial blood gas often reveals respiratory acidosis, hypoxia and hypercapnia due to hypoventilation resulting in carbon dioxide retention, hypoxemia and associated lactic acidosis [4]. Chest radiograph may show cardiomegaly and pleural effusions [1]. It is known that the thyroid hormone plays an important role in the heart and vascular system in which hyperthyroidism and hypothyroidism affect cardiac contractility, myocardial oxygen consumption, cardiac output, blood pressure, and systemic vascular resistance as well as arrhythmias such as atrial fibrillation [5]. Pleural effusions can be seen with hypothyroidism but usually as a result of secondary process such as pericardial fluid, congestive heart failure or ascites. This is termed hypothyroid-associated pleural effusions. Typical hypothyroid pleural effusions tend to be small, non-inflammatory and have characteristics between transudates and exudates. They are thought to result from pulmonary or pleural capillary leak [6]. Both cardiomyopathy and pleural effusions are usually reversible with thyroid hormone replacement therapy.

Unlike the aforementioned expected findings, this case was characterized by severe hypokalemia, metabolic alkalosis, low normal glucose and normal cortisol. There was no pleural effusion or cardiomegaly on chest radiograph (Fig. 4). Serum troponin was $0.01 \mathrm{ng} / \mathrm{mL}$ and electrocardiogram (ECG) (Fig. 2) did not show signs of cardiac ischemia. Notably, hyponatremia was absent. Generally, myxedema coma results from several years of untreated hypothyroidism, and it carries a greater mortality than $50 \%$ [4]. In this case, the family stated that her symptoms of lethargy and medical non-compliance with levothyroxine started about 6 months ago. Although a respiratory acidosis was expected due to her chronic regimen of morphine extended-release $15 \mathrm{mg}$, arterial blood gas (Table 1) on admission showed $\mathrm{pH}$ of 7.63, $\mathrm{PaCO}_{2}$ of $39.6 \mathrm{~mm} \mathrm{Hg}, \mathrm{PaO}_{2}$ of $56 \mathrm{~mm} \mathrm{Hg}$ and a bicarbonate of $41.9 \mathrm{mmol} / \mathrm{L}$, reflecting a primary metabolic alkalosis without respiratory compensation. In addition to severe hypokalemia of $2.0 \mathrm{mmol} / \mathrm{L}$ and hypochloremia of $78 \mathrm{mmol} / \mathrm{L}$, metabolic alkalosis was thought to be due to contraction alkalosis from dehydration relating to lack of oral hydration. The patient was not on diuretic therapy. Hypochloremia, caused by chloride loss from the gut, kidney, skin, or in this case, decreased oral intake results in alkalosis because bicarbonate generated during the production of gastric acid returns to the circulation leading to net accumulation of base with net loss of acid from the extracellular fluid (ECF) [7]. Hypokalemia augments the alkalosis by causing an intracellular shift of hydrogen, thus increasing ECF bicarbonate concentrations and potassium depletion independently augments bicarbonate reabsorption in the proximal tubule [7]. Contraction alkalosis, defined as acute decrease in ECF [8], further maintains alka-

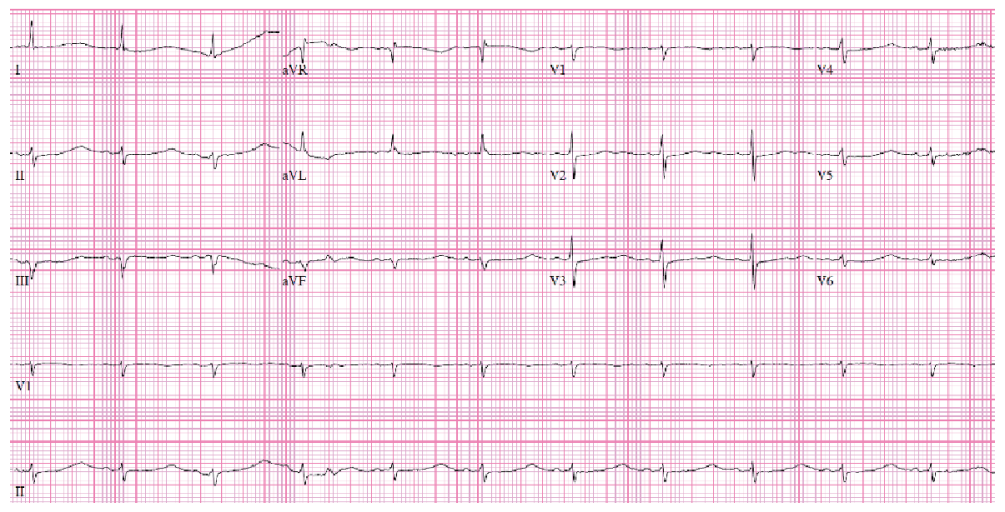

Figure 2. ECG showing ventricular rate 71/min, QTc: $633 \mathrm{~ms}$, normal sinus rhythm (NSR), T wave flattening and prolonged QT. 
Table 1. Relevant BMP/CBC/Thyroid Labs Examination at Admission

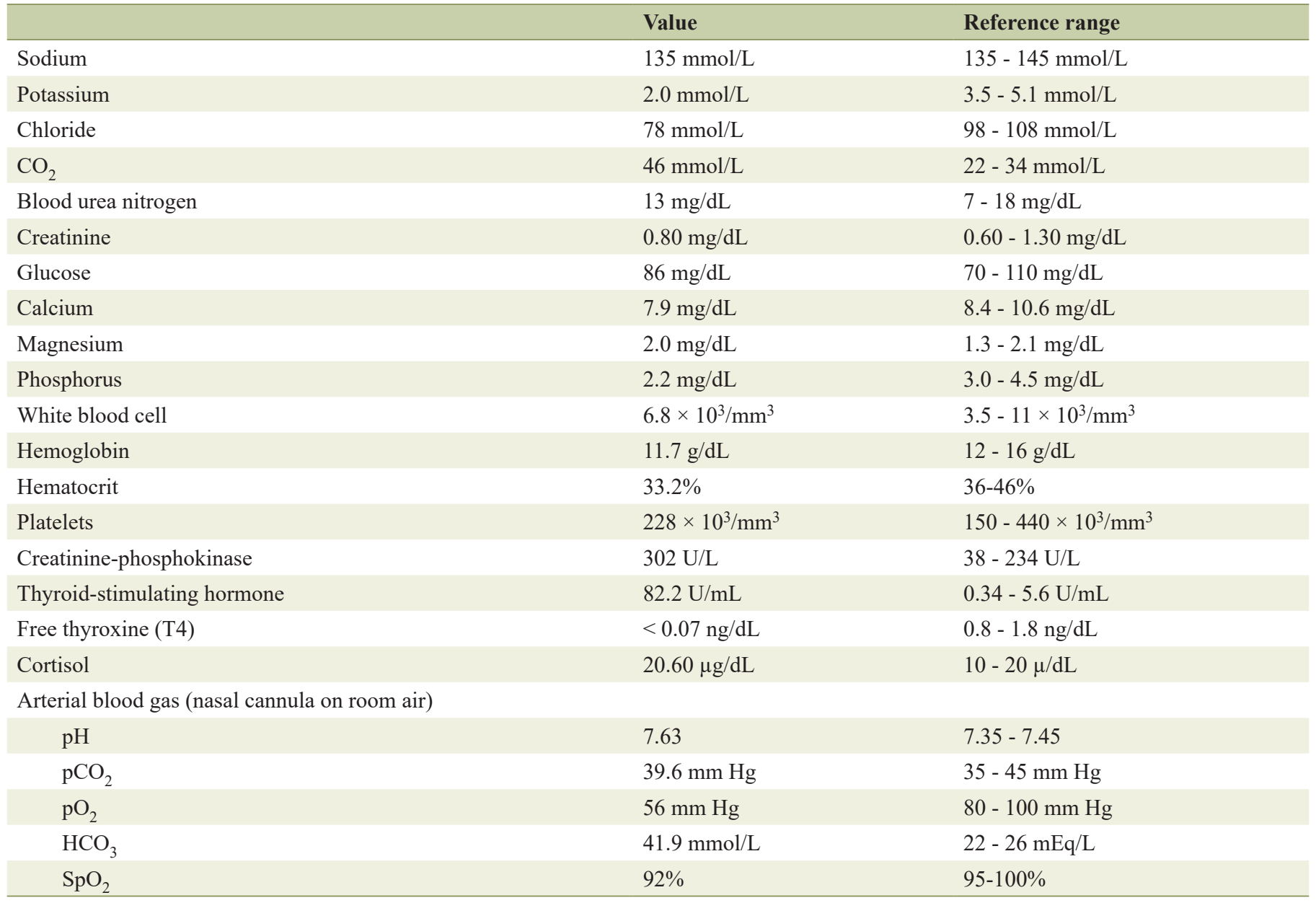

losis by increased fluid reabsorption in the proximal tubule where bicarbonate is preferentially reabsorbed compared with chloride [7].

Although a compensatory respiratory acidosis was expected, the arterial blood gas was more consistent with respiratory alkalosis. Some common causes for primary respiratory alkalosis include salicylate poisoning, encephalitis, head trauma, sepsis, hyperventilation and pulmonary embolism [9-11]. As mentioned earlier, patients with severe hypothyroidism can present with respiratory acidosis due to $\mathrm{CO}_{2}$ retention from respiratory muscle fatigue and impaired ventilatory drive to hypoxia and hypercapnia [12]. In this case, the primary metabolic alkalosis seemed to overshadow possible respiratory acidosis. Furthermore, chronic severe hypothyroidism may be associated with a diminished basal metabolic rate and lower $\mathrm{CO}_{2}$ production [12] thereby exacerbating her alkalosis. Salicylate poisoning was ruled out as her serum salicylate was below 1 $\mathrm{mg} / \mathrm{dL}$.

Another interesting abnormality on labs is the finding of elevated creatinine-phosphokinase (CPK) of 302 U/L. Myopathy is a known complication of long-standing hypothyroidism and the incidence of musculoskeletal symptoms varies from $30-80 \%[13,14]$. Specifically, a majority of patients with hypothyroidism have been shown to have an increased serum CPK $[1,15]$. This phenomenon was observed as early as 1897 , termed Hoffman's syndrome, whereby thyroidectomy in a patient caused proximal weakness, myopathy and pseudohypertrophy of muscles [16]. In general, the elevation of CPK in hypothyroidism is proportional to the degree of the hypothyroid state [14]. While the exact etiology of the myopathy is unknown [13], authors describe an increase in type I muscle fiber and selective atrophy of type II fibers, together with low mitochondrial enzyme activities [14]. Current opinions support the theory that thyroxine (T4) deficiency leads to abnormal glycogenolysis and mitochondrial oxidative metabolism, which in turn impairs muscle function as reflected in selective atrophy of type II fibers (fast twitch), which are more dependent on glycolysis for their energy supply. Type I (slow twitch) hypertrophy may be compensatory [17]. With severe or prolonged oxidative damage, muscle cell injury and rhabdomyolysis may occur leading to changes in muscle cell membrane permeability and increase in serum CPK [1]. This results in slowness of muscle contraction. Delayed relaxation of deep tendon reflexes is due to impaired calcium sequestration by sarcoplasmic reticulum, which prolongs twitch duration [16]. Altogether, delayed tendon reflex is a common clinical finding 


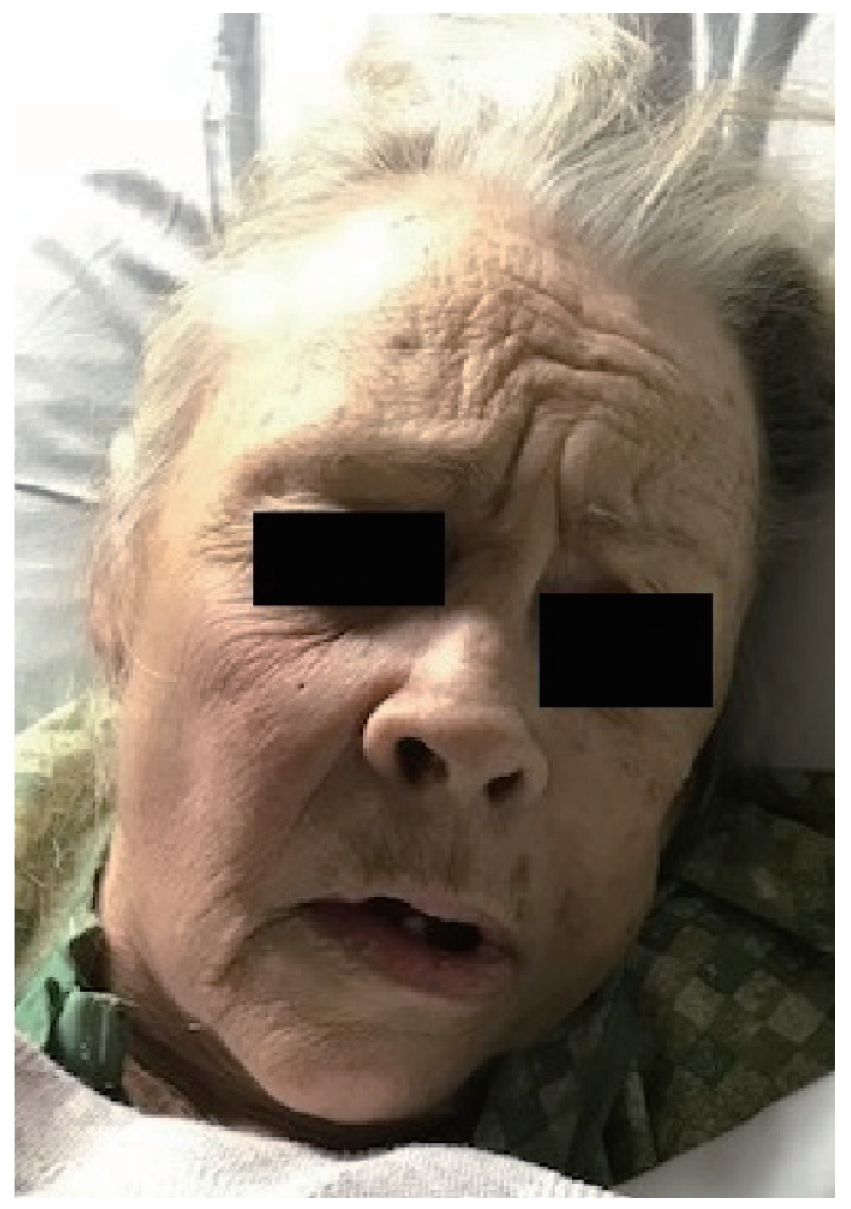

Figure 3. Hypothyroid facies, dry skin, coarse hair, saddle nose, periorbital edema, ptosis and apathetic appearance.

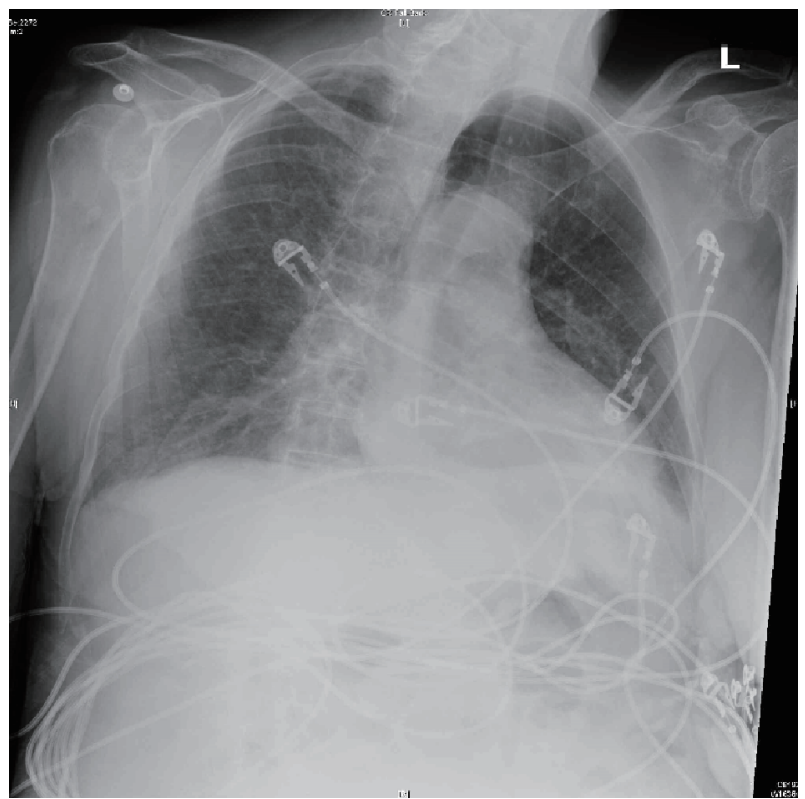

Figure 4. No pleural effusion or cardiomegaly on chest X-ray.

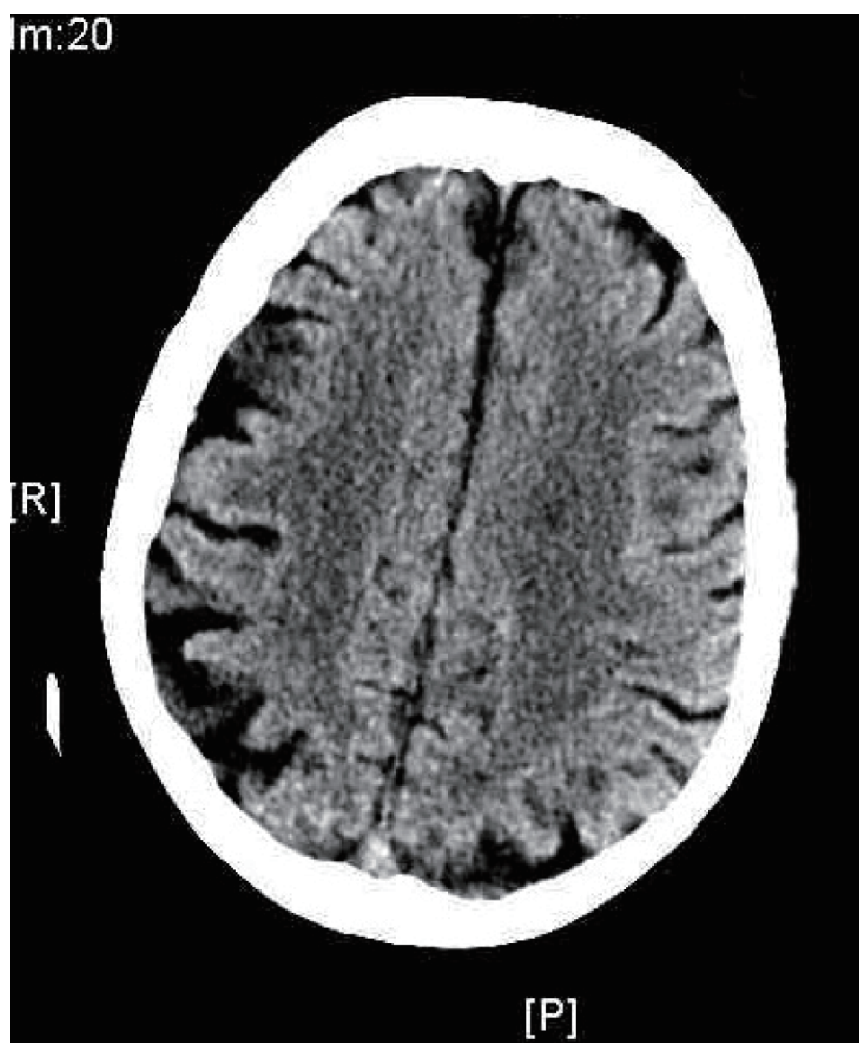

Figure 5. Brain CT showing cortical atrophy.

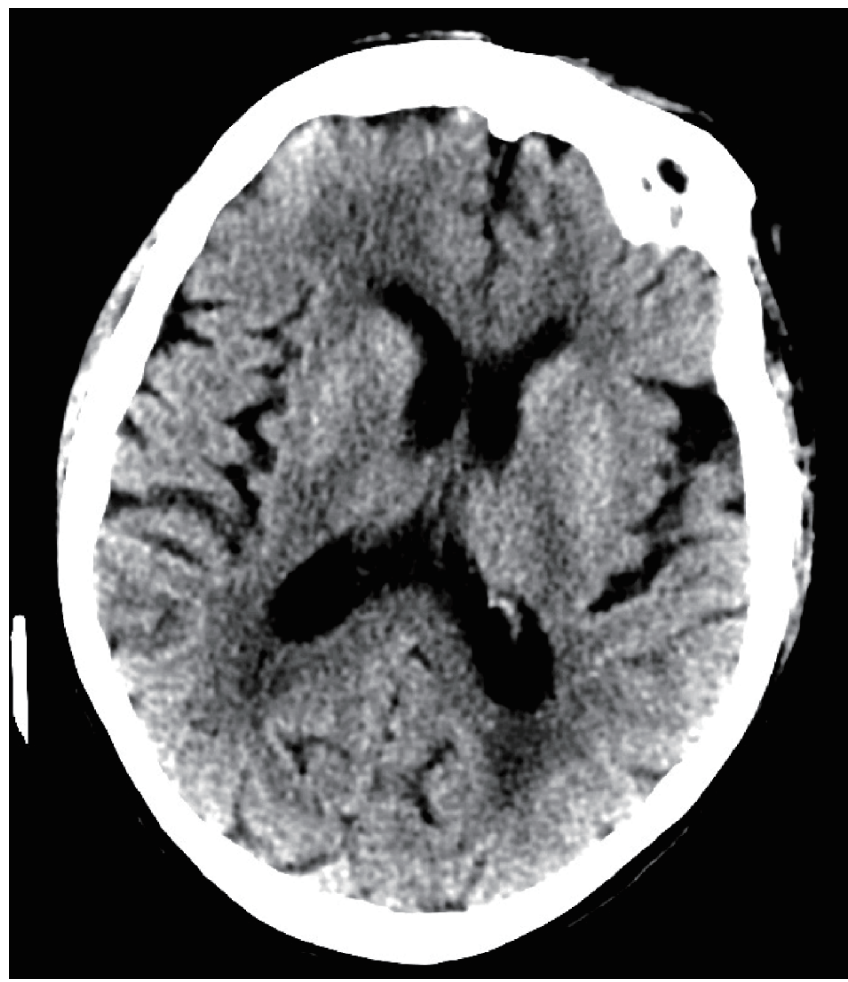

Figure 6. Brain CT showing prominent sulci. 
on physical exam in hypothyroidism [1].

\section{Conclusions}

This case report illustrates the importance of checking for thyroid dysfunction especially in the elderly who present with altered mental status and cachexia. If it is not possible to obtain an accurate history, it is best to order thyroid function studies as most of the symptoms associated with hypothyroidism or myxedema coma may be reversible [5] with prompt institution of appropriate therapy [4]. In this case, the myxedema coma may have triggered an acute worsening of her mental status on her baseline dementia. Brain CT (Figs. 5, 6) showed prominent sulci, particularly around the ventricular system as well as loss of convexity indicating generalized atrophy. Evidence of brain atrophy is consistent with the patient's history of dementia [18] which may have been a precipitating factor in her non-compliance with levothyroxine leading to severe hypothyroidism and myxedema coma. In this case, despite timely diagnosis and appropriate treatment, the family eventually elected for palliative/hospice care due to the patient's severely decompensated state which did not respond to appropriate medical therapy.

\section{Acknowledgments}

We would like to extend our gratitude to the Palliative Care Team at Ocala Regional Medical Center consisting of Dr. Raju Dantuluri and ARNP Ms. Lori Williams in assisting the family of the patient to make decisions regarding hospice care.

\section{Financial Disclosure}

This research was supported (in whole or in part) by HCA and/ or an HCA affiliated entity. The views expressed in this publication represent those of the author(s) and do not necessarily represent the official views of HCA or any of its affiliated entities.

\section{Conflict of Interest}

The authors have no conflict of interest to declare

\section{Informed Consent}

Written informed consent was obtained from the patient for the publication of this case report along with the accompanying images.

\section{Author Contributions}

Jake Namjik Cho: data curation (obtained the relevant clinical labs and imaging), formal analysis (applied clinical analysis to the labs, ECG and imaging), investigation (conducted research and investigation into discussion topics) validation (verification of the facts in the case report, verification of references) and writing (preparation of the original draft, review and editing of the manuscript). Stephen Avera: formal analysis (assisted in the interpretation of the clinical labs), validation (verification of facts presented in the case report, verification of references) and writing (reviewed and edited the section topic of metabolic alkalosis). Kenneth Iyamu: validation (verification of the facts in the case report, verification of references) and writing (reviewed and edited the manuscript, in particular the section of treatment and discussion of palliative care).

\section{References}

1. Wall CR. Myxedema coma: diagnosis and treatment. Am Fam Physician. 2000;62(11):2485-2490.

2. Mathew V, Misgar RA, Ghosh S, Mukhopadhyay P, Roychowdhury P, Pandit K, Mukhopadhyay S, et al. Myxedema coma: a new look into an old crisis. J Thyroid Res. 2011;2011:493462.

3. Eledrisi MS. Myxedema coma or crisis workup. Oct 10, 2018. Medscape. https://emedicine.medscape.com/ article/123577-workup. Accessed 02/12/2018.

4. Newmark SR, Himathongkam T, Shane JM. Myxedema coma. JAMA. 1974;230(6):884-885.

5. Klein I, Danzi S. Thyroid disease and the heart. Circulation. 2007;116(15):1725-1735.

6. Gottehrer A, Roa J, Stanford GG, Chernow B, Sahn SA. Hypothyroidism and pleural effusions. Chest. 1990;98(5):1130-1132.

7. Galla JH. Metabolic alkalosis. J Am Soc Nephrol. 2000;11(2):369-375.

8. Luke RG, Galla JH. It is chloride depletion alkalosis, not contraction alkalosis. J Am Soc Nephrol. 2012;23(2):204207.

9. Al-Jaghbeer M, Kellum JA. Acid-base disturbances in intensive care patients: etiology, pathophysiology and treatment. Nephrol Dial Transplant. 2015;30(7):1104-1111.

10. Goldhaber SZ, Elliott CG. Acute pulmonary embolism: part I: epidemiology, pathophysiology, and diagnosis. Circulation. 2003;108(22):2726-2729.

11. Williams RH, Erickson T. Evaluating acetaminophen and salicylate poisoning in an emergency setting. Laboratory Medicine. 1998;29(1):33-37.

12. Lee HT, Levine M. Acute respiratory alkalosis associated with low minute ventilation in a patient with severe hypothyroidism. Can J Anaesth. 1999;46(2):185-189.

13. Jonderko G, Smrek M. [Correlation between creatine kinase activity and magnesium level in the serum in thyroid function disorders]. Pol Tyg Lek. 1977;32(44):17051706.

14. Kung AW, Ma JT, Yu YL, Wang CC, Woo EK, Lam KS, Huang CY, et al. Myopathy in acute hypothyroidism. Postgrad Med J. 1987;63(742):661-663.

15. Ranka R, Mathur R. Serum creatine phosphokinase in thyroid disorders. Indian J Clin Biochem. 2003;18(1):107- 
110.

16. Mangaraj S, Sethy G. Hoffman's syndrome - A rare facet of hypothyroid myopathy. J Neurosci Rural Pract. 2014;5(4):447-448.

17. McKeran RO, Slavin G, Andrews TM, Ward P, Mair WG.
Muscle fibre type changes in hypothyroid myopathy. J Clin Pathol. 1975;28(8):659-663.

18. Degnan AJ, Levy LM. Neuroimaging of rapidly progressive dementias, part 1: neurodegenerative etiologies. AJNR Am J Neuroradiol. 2014;35(3):418-423. 